\title{
IMPLEMENTAÇÃO DO ENTERPRISE RESOURCE PLANNING EM UMA METALÚRGICA COM LINHAS DE PRODUTOS DIFERENCIADAS
}

\author{
Annibal Affonso Neto \\ annibal@terra.com.br \\ Universidade de Brasília - UnB, \\ Brasília, Distrito Federal, Brasil.
}

\section{Clovis Neumann}

clovisneumann@unb.br Universidade de Brasília - UnB, Brasília, Distrito Federal, Brasil.

\section{RESUMO}

Destaques:

O estudo contemplou a implementação do Enterprise Resource Planning (ERP) na estratégia de produção e no desempenho.

O comportamento das variáveis determinantes da estratégia de produção foi analisado.

Os reflexos da adoção do ERP na estratégia de produção foram estudados.

A adoção do ERP trouxe melhorias para a operação da empresa, sobretudo na confiabilidade e rastreabilidade da informação.

Objetivo: analisar o impacto da implementação do ERP na estratégia de produção e no desempenho de uma metalúrgica de médio porte sediada no Distrito Federal e com unidade em Goiás.

Desenho/Metodologia/Abordagem: estudo de caso único, com o intuito de compreender em profundidade os diversos aspectos associados à implantação do ERP em uma média indústria. $O$ trabalho considerou as variáveis determinantes da estratégia de produção e foi desenvolvido nas três unidades de negócios da metalúrgica: portas e janelas, perfilados, postes e braços, considerando a percepção dos colaboradores. Do ponto de vista metodológico, foi elaborado a partir de pesquisa qualitativa nas modalidades pesquisa documental, observação e estudo de caso único instrumental, já que a finalidade foi compreender determinado fenômeno no contexto em que ocorreu. A pesquisa documental possibilitou o levantamento de informações que subsidiaram a elaboração do roteiro de entrevistas e a identificação do público-alvo.

Resultados: foram identificados os reflexos da adoção do ERP na estratégia de produção. Para avaliar o impacto na produção, foram considerados os objetivos de desempenho operacional. Com base no estudo, concluiu-se que a adoção do ERP trouxe melhorias para a operação da empresa, sobretudo na confiabilidade e rastreabilidade da informação.

Limitações da investigação: como a implantação era recente, muitos colaboradores ouvidos ainda não possuíam uma ideia clara das mudanças e do alcance que a adoção do ERP traria para as fábricas e a produção.

Implicações práticas: o estudo permitiu o levantamento e registro dos desafios na implantação do ERP em uma metalúrgica com linhas de produtos diferenciadas, de onde advém a sua originalidade e relevância.

Originalidade/valor: a originalidade consiste no fato de o estudo ter sido realizado em uma metalúrgica de médio porte, localizada na região centro-oeste, com três unidades e linhas de produtos diferenciados, quando a implantação do ERP estava sendo concluída.

Palavras-chave: Planejamento; Informações; Gerenciais; Estratégia; Produção. 


\section{INTRODUÇÃO}

O estudo Avaliação do Desempenho do Brasil Mais Produtivo, elaborado pela Comissão Econômica para a América Latina e o Caribe e pelo Instituto de Pesquisa Econômica Aplicada (CEPAL; IPEA, 2018), mostra que a trajetória da produtividade na indústria brasileira é caracterizada por uma estagnação nas últimas décadas. Segundo o estudo, metade das diferenças na renda per capita entre nações pode ser explicada por diferenças de produtividade. Na mesma linha de estudo, o Banco Interamericano de Desenvolvimento revela que as baixas taxas de crescimento da produtividade são o principal entrave ao crescimento econômico na América Latina.

Nos últimos anos, muito foi debatido com relação a fatores externos à fábrica, notadamente reformas, que meIhorem o ambiente de negócios, a educação profissional e a infraestrutura, e simplifiquem a regulação e a tributação.

Internamente à fábrica, em razão da necessidade de serem competitivas aumentando a produtividade diante da concorrência global, as empresas brasileiras vêm investindo na implantação do Enterprise Resources Planning (ERP). Muitas iniciam o processo de implantação sem ter a noção exata dos desafios e suas implicações. Isso, porque não se trata apenas de implantar mais um sistema e sim realizar uma transformação organizacional com consequências para a empresa como um todo.

Nesse contexto, o presente estudo tem por objetivo analisar a implantação do ERP em uma empresa metalúrgica de médio porte, com linhas de produtos diferenciados, analisando as repercussões da adoção do software na estratégia de produção a partir dos objetivos de desempenho operacional.

No desenvolvimento da pesquisa, adotou-se uma abordagem de pesquisa diferente da linha que vem dominando os enfoques adotados por pesquisadores em artigos publicados em periódicos conceituados nacional e internacionalmente, que é o de estudar o tema 'estratégia de produção' visando relacionar a influência e o alinhamento das prioridades competitivas às decisões estruturais e infraestruturais. As conclusões retratam os benefícios obtidos pela empresa - objeto do estudo - com a implantação do ERP.

\section{REFERENCIAL TEÓRICO}

\section{Enterprise Resource Planning}

Os Enterprise Resource Planning (ERP), também chamados de Sistemas Integrados de Gestão, foram desenvolvidos no final dos anos 1990, como uma solução e evolução do Materials Requirement Planning (MRP) e MRP II (Ferreira et al., 2010; Oleskow et al., 2002; Rashid et al., 2002), passando a ser utilizados pelas empresas no mundo todo.

Seu desenvolvimento e adoção por parte das empresas emergiu da necessidade de integrar informações que se encontravam dispersas, em um grande número de outros sistemas, o que tornava difícil o acesso a essa informação, sem contar o problema decorrente de dados divergentes entre os vários sistemas disponíveis.

Os ERP propiciam uma fonte de dados unificada para todas as atividades da empresa e, portanto, representam a espinha dorsal do processo de gestão das informações. Essa gestão possibilita uma melhoria do processo decisório e contribui para torná-lo consistente, oportuno e confiável em unidades organizacionais e localizações geográficas (Chatzoglou et al., 2016). Os ERP integram as informações dos setores da empresa utilizando o conceito de base de dados única, possibilitando a melhoria contínua dos processos e atendendo a necessidade de informações confiáveis e em tempo real (Laudon; Laudon, 2007).

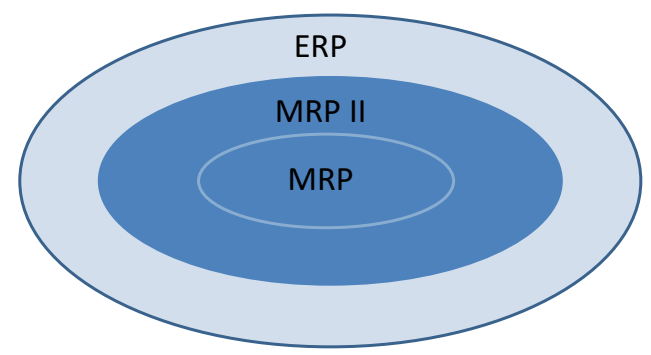

Figura 1. ERP como uma evolução do MRP Fonte: Os próprios autores

Um ERP é, portanto, um software utilizado para o planejamento dos recursos empresariais por integrar as funções empresariais de forma a possibilitar operações mais eficientes. Representam uma integração de informações contábeis, financeiras, de produção e compras entre outras. Devido à arquitetura aberta, é viável a sua integração com outros sistemas operacionais, bancos de dados e plataformas de hardware.

A Figura 2 apresenta a estrutura de um ERP e seus respectivos módulos que possibilitam a integração de diversas informações da empresa.

Segundo Barth e Koch (2019), nos últimos anos, a penetração dos sistemas de planejamento de recursos empresariais, $E R P$, nas pequenas, médias e grandes empresas aumentou. As organizações são demandadas a adaptar seus sistemas e realizar atualizações de ERP para reagir a ambientes de ne- 


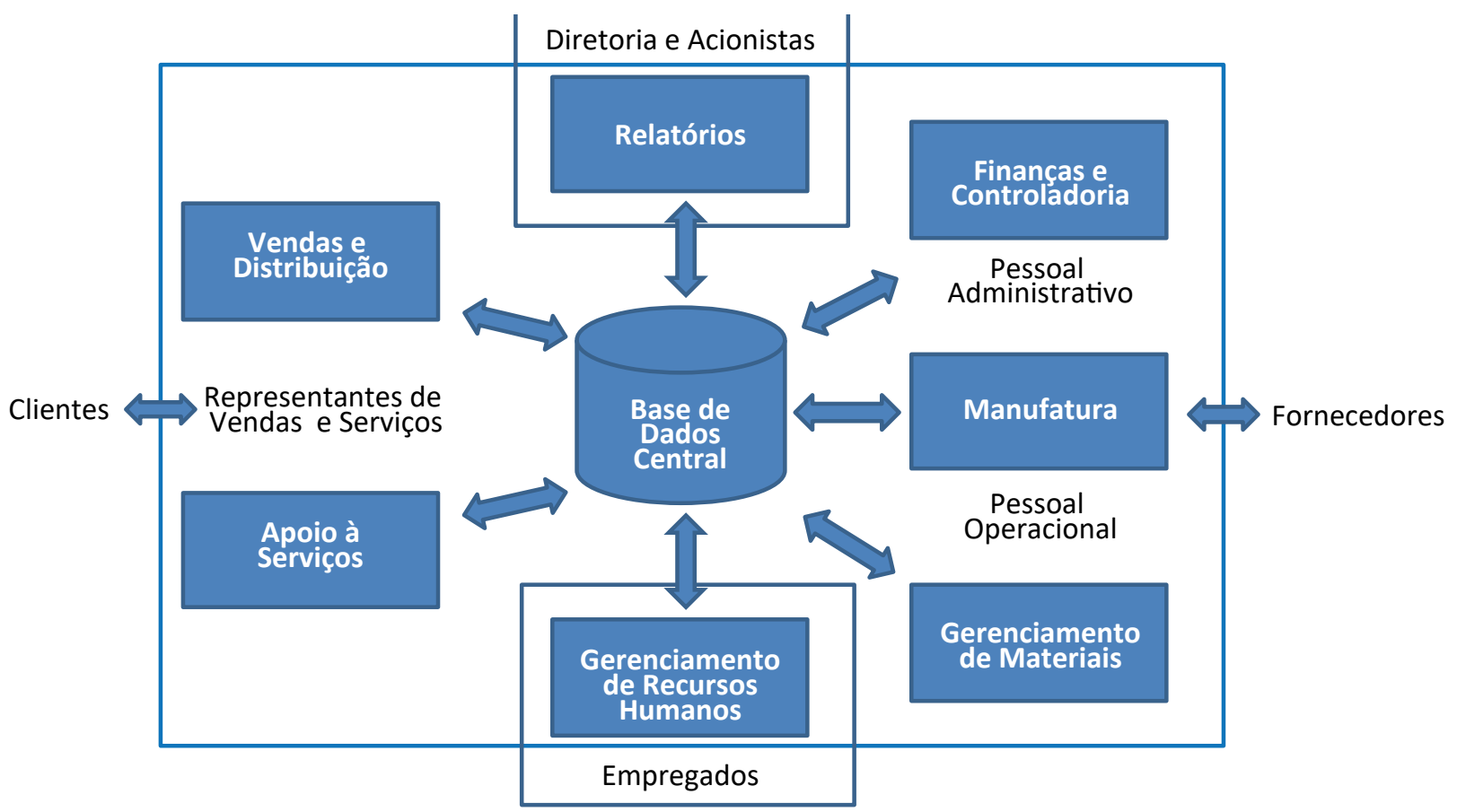

Figura 2. Enterprise Resource Planning

Fonte: Adaptado de Davenport, 1998; Junior 2008

gócios em rápida mudança, aprimoramentos tecnológicos e pressão crescente da concorrência.

O ERP tradicional nas organizações está cada vez mais impactado pelas tecnologias de informação emergentes, como serviços em nuvem e tecnologias de mídia social. Uma pesquisa recente realizada pelo grupo Gartner, em 2013, revela que $47 \%$ das organizações planejam mudar para sistemas baseados em nuvem nos próximos cinco anos (Rayner, 2014).

\section{Implementação do ERP}

Em que pese os desafios envolvidos na implantação do Planejamento de Recursos Empresariais, sobretudo para as empresas de menor porte e com menos recursos disponíveis, muitas empresas estão optando por implantá-los, sobretudo por causa das frustrações com sistemas incompatíveis, principalmente para tornar os processos empresariais mais ágeis. Alguns fatores, segundo Padilha e Marins (2005), são fundamentais para o sucesso na implantação do $E R P$, quais sejam:

- obter o comprometimento da alta direção (Commitment);

- implementar o gerenciamento de mudanças objetivando mitigar o "medo" dos usuários pouco informados;
- identificar os usuários-chave, que são pessoas fundamentais em seus respectivos setores;

- $\quad$ escolher para Gerente do Projeto (Gerente-Usuário) um profissional experiente e respeitado, de forma a descaracterizar o ERP como um sistema da área de informática, e sim como um redesenho do modelo de gestão;

- $\quad$ planejar e realizar treinamentos;

- definir os papéis e responsabilidades na implementação do sistema, por meio da união de conhecimentos e esforços;

- customizar o sistema à empresa e vice-versa, refletindo sobre a realidade atual da empresa e a utilização das melhores práticas (best-practices);

- $\quad$ escolher e contratar a consultoria adequada (know-how);

- $\quad$ assegurar a qualidade (quality assurance);

- $\quad$ simplificar em todos os aspectos, desde a definição de modelos, passando pelo desenho da solução, até na própria implementação do sistema. 
Ainda que a adoção de alguns cuidados possa mitigar os riscos inerentes à implantação de um $E R P$, a tarefa não é trivial. Segundo os autores supramencionados, as dificuldades com a implantação do ERP decorrem fundamentalmente de dois fatores:

- A empresa não fez as escolhas estratégicas necessárias para configurar os sistemas e os processos;

- O processo de implementação escapa ao controle da empresa naturalmente.

Com relação ao nível de controle das operações que o $E R P$ deve proporcionar à empresa, esse é dependente do projeto do fluxo de produtos e serviços existentes. Uma cadeia de valor bem elaborada contempla diversos controles e outras funções nos processos da empresa de maneira muito mais simples e com custos mais acessíveis com o ERP.

Estudos sugerem que $60 \%$ dos projetos de ERP fracassam por diversos fatores, como: o retorno aquém do esperado, a incapacidade do sistema para atender a determinados requisitos funcionais, as limitações de orçamento, manutenção e custo de treinamentos elevados, a estimativa inadequada de prazos, o funcionamento incorreto do sistema e seu não atendimento às expectativas iniciais (Ghosh, 2012).

Essas falhas, quando ocorrem, podem ser atribuídas à:

- falta de clareza sobre os requisitos funcionais. As funcionalidades muitas vezes não são bem especificadas;

- falta de compromisso da gestão. A cúpula da empresa decide implantar o ERP e, por não ter a noção exata da dimensão e as peculiaridades do projeto, acaba não se comprometendo;

- formação inadequada. Os usuários não são devidamente treinados;

- seleção inadequada do pacote de software. Os pacotes são escolhidos em função da experiência dos empregados quando da implantação do ERP nas empresas em que trabalhavam anteriormente;

- mau dimensionamento de prazos e expectativas. 0 tempo e o esforço necessários para a implementação são subestimados;

- incompatibilidade com os processos de negócios. 0 desalinhamento com os processos de apoio pode afetar o desempenho do ERP.
Um bom planejamento do processo de implantação, desde a escolha do fornecedor, é fundamental para que se obtenham os resultados esperados. Scheckenbach et al. (2014, p. 659) descrevem as atualizações de ERP como "principalmente destinadas a aproveitar as novas tecnologias e estratégias de negócios para garantir que a organização acompanhe as tendências de desenvolvimento de negócios".

Em um dos modelos mais recentes, Peng e Nunes (2017) propõem a estrutura de avaliação do $9 D E R P$, que inclui nove dimensões e 85 critérios de avaliação, com foco em fatores críticos de falha a serem abordados na pós-implementação.

Huang e Yokota (2019) publicaram uma pesquisa cujos resultados indicam que a utilização do modelo de avaliação de alinhamento entre negócios e ERP é um método mais conveniente para ajudar as organizações a identificar o status do alinhamento entre organizações e sistemas de ERP.

\section{Estratégia de produção}

As atividades da manufatura ou as prioridades estratégicas foram identificadas por Skinner (1969) como sendo: produtividade, serviço, qualidade e retorno sobre o investimento.

Já a estratégia de produção pode ser estudada de diversos pontos de vista, de acordo com Skinner (1969), a respeito da importância da produção para a estratégia da empresa (Cohen; Lee, 1985; Swamidass; Newell, 1987; Anderson et al., 1989, Amoako-Gyampah; Boye, 2001). Segundo Garvin (1993), a maioria das publicações aborda quatro principais prioridades competitivas: custo, qualidade, entrega e flexibilidade. A essas quatro prioridades competitivas, o autor acrescenta mais uma que denominada de serviços.

Slack et al. (2010) identificam cinco objetivos de desempenho operacional que fazem parte de todos os tipos de operações, que são: qualidade, flexibilidade, velocidade, confiabilidade e custo.

O objetivo da estratégia de produção é o de prover um padrão de decisões consistentes no que tange ao processo de produção, e disponibilizar para a empresa uma orientação em relação à maneira de empregar os recursos de forma a sustentar uma vantagem competitiva no longo prazo. Identificadas as prioridades competitivas, é possível desenvolver o design do sistema de produção por intermédio de um conjunto de fatores relacionados às áreas de decisão. A estratégia da produção, portanto, consiste no estabelecimento de políticas e planos para utilizar os recursos de uma empresa, objetivando uma melhor sustentação da estratégia competitiva em longo prazo. 
Segundo Slack (2005), durante muito tempo considerou-se haver contradição no conceito de estratégia de produção/operações, sendo a primeira uma abordagem externa e de longo prazo focada na agregação de valor e de responsabilidade do principal executivo, e a segunda, estratégia de operação, se refere à algo complexo, detalhado, relativo ao dia a dia da empresa e de responsabilidade dos níveis hierárquicos inferiores.

Segundo Okoshi et.al. (2019), a estratégia de fabricação tem um papel importante na estratégia competitiva dos negócios, porque conecta os indicadores de desempenho aos objetivos da empresa. A estratégia de operações é organizada em objetivos de desempenho e áreas de decisão que definem seu conteúdo.

As estratégias de produção são desenvolvidas, considerando os critérios competitivos ou objetivos de desempenho operacional, que permitem uma análise mais adequada acerca do posicionamento dos produtos e bens frente às exigências do mercado e dos clientes.

Comumente são utilizados quatro objetivos de desempenho operacional básicos: custos, qualidade, entrega e flexibilidade. Paiva et al. (2009) identificam cinco critérios competitivos na área da administração da produção, que se relacionam com a estratégia de negócios da organização, a saber: custos, qualidade, desempenho de entrega, flexibilidade e inovatividade. O último critério, a inovatividade, "é tradicionalmente definido como a habilidade da empresa em lançar novos produtos e/ou serviços em curto espaço de tempo" (Paiva et al., 2009, p. 56).

As empresas estão desenvolvendo novos modelos de gerenciamento para suas funções de fabricação. A complexidade dos padrões competitivos e a mudança de paradigma com base na manufatura conectada exigem uma compreensão mais próxima das relações entre os resultados de desempenho e as capacidades de fabricação das empresas (Nudurupati et al., 2016; Lauras et al., 2010).

Segundo Hill (2005), a formulação da estratégia de produção contempla cinco etapas:

- definição dos objetivos empresariais;

- determinação das estratégias de marketing para alcançar esses objetivos;

- $\quad$ avaliação sobre a forma com a qual diferentes produtos conquistam mercados em detrimento dos concorrentes;

- estabelecimento da forma mais adequada para disponibilizar esses produtos;
- $\quad$ promoção da infraestrutura requerida para suportar as operações.

Os estudos que conectam a estratégia de produção aos resultados de desempenho geralmente exploram os efeitos de causalidade entre políticas e resultados. Esmaeel et al. (2018) mostram como a estratégia de produção alinha e conecta os resultados às decisões estratégicas de fabricação.

\section{Implementação do ERP, estratégia de operações e objetivos de desempenho operacional}

O relacionamento entre a estratégia de negócios, estratégia de produção e a estratégia de sistemas de informação vem sendo analisada na literatura. Diversas pesquisas foram publicadas abordando os ERP e o impacto da sua implementação nas organizações (Valipour et al, 2012; Chen et al., 2009; Dantes; Hasibuan, 2011).

Cao e Dowlatshahi (2005), por exemplo, identificaram vínculos entre a estratégia de produção e a orientação estratégica de sistemas de informação. Eles analisaram a correlação entre o desempenho da empresa e os sistemas de informação, quando do alinhamento entre a estratégia de produção e a estratégia de sistema de informações (Byrd et al. 2006; Chan; Reich, 2007).

Sabherwal e Chan (2001) e Chan (2002) estudaram a correlação entre a orientação estratégica de sistemas de informação e as decisões em termos de estratégia de operações, da mesma forma que entre a estratégia de negócios e a estratégia de informações no desempenho do negócio.

Outra pesquisa abordou a implementação do ERP na Rolls-Royce, utilizando a metodologia do estudo de caso focando principalmente o projeto de implementação, os aspectos culturais envolvidos e os problemas técnicos da implementação (Yusuf et al., 2004).

O relacionamento da implantação do ERP com a estratégia e aspectos culturais envolvidos em alinhar a implementação do ERP a produtos e processos também foi abordado por Bowersox et al. (1998), Davenport (1998), Hammer e Stanton (1999), Jacobs e Whybark (2000) e Soh et al. (2000).

Outros autores comtemplaram a implantação do ERP em manufatura (Yen; Sheu, 2004; Yusuf et al., 2004) e nas prioridades competitivas ou objetivos de desempenho operacional (Yen; Sheu, 2004). Esse estudo concluiu que a implementação do ERP impacta diversos aspectos da operação do negócio e do desempenho. 


\section{MÉTODO}

O presente estudo é de natureza exploratória. A pesquisa na metalúrgica se desenvolveu por intermédio da pesquisa qualitativa nas modalidades pesquisa documental, observação e estudo de caso único instrumental.

Optou-se pela pesquisa qualitativa por se desenvolver no ambiente natural, e pelo fato de a preocupação do investigador ser o significado que as pessoas dão aos acontecimentos e o enfoque dedutivo. A pesquisa documental foi desenvolvida em particular na página da empresa na internet e em manuais e procedimentos utilizados pelos colaboradores.

O objetivo da primeira etapa da investigação foi conhecer o histórico da metalúrgica, desde a fundação na década de 1960, e as principais transformações que ocorreram. Em seguida, desenvolveu-se o estudo de caso nas unidades em que as técnicas aplicadas foram a observação e a entrevista.

$O$ objeto do estudo foi a implantação do $E R P$. Foi utilizado o estudo de caso único instrumental já que a finalidade da pesquisa foi compreender, em profundidade e de diversos pontos de vista, determinado fenômeno no contexto em que ocorreram.

\section{No estudo de caso instrumental:}

“O interesse no caso deve-se à crença de que ele poderá facilitar a compreensão de algo mais amplo, uma vez que pode servir para fornecer insights sobre um assunto ou para contestar uma generalização amplamente aceita, apresentando um caso que nela não se encaixa" (Mazzoti, 2006, p. 641).

De acordo com Yin (2005, p. 33), o estudo de caso "é o método escolhido ao se analisar acontecimentos contemporâneos quando não se podem manipular comportamentos relevantes". $O$ autor acrescenta:

“É uma forma de se fazer pesquisa empírica que
investiga fenômenos contemporâneos dentro de
seu contexto de vida real, em situações em que
as fronteiras entre o fenômeno e o contexto não
estão claramente estabelecidas, onde se utiliza
múltiplas formas de evidência”.

Para a coleta de informações nas entrevistas, foi utilizada a metodologia da Consulta-Entrevista, a partir da qual seleciona-se um grupo de especialistas a serem entrevistados para debater um determinado assunto. $O$ procedimento da
Consulta-Entrevista consiste em, para o primeiro entrevistado, elaborar um roteiro de perguntas adequadas às características do indivíduo. Esse conjunto de questões será o roteiro para a entrevista com os demais entrevistados. Feita a primeira entrevista, realiza-se uma síntese da discussão, que serve de diretriz para a elaboração do questionário para o segundo entrevistado. Na segunda entrevista, procura-se corroborar as informações obtidas na primeira entrevista e complementar o conhecimento sobre o assunto. Repete-se esse processo para os demais entrevistados até que as informações obtidas em entrevistas subsequentes não apresentassem novo conhecimento. A metodologia possibilitou alcançar um conhecimento amplo do assunto, a partir do conhecimento parcial, disperso e geralmente tendencioso dos colaboradores.

\section{RESULTADOS}

\section{Perfil do grupo empresarial e dos respondentes}

A empresa estudada é uma metalúrgica, líder no segmento na região Centro-Oeste, que atua na fabricação e comercialização de, entre outros, perfilados, tubos industriais, chapas e laminados. Possui uma fábrica com unidades de negócios no Distrito Federal e no estado de Goiás, e diversas lojas que comercializam produtos fabricados pela indústria, produtos agroindustriais, ferramentaria, acessórios de serralheria e equipamentos de proteção individual.

A empresa possui três unidades de negócios: portas e janelas; perfilados; postes e braços. A unidade de perfilados contempla duas plantas: uma em Brasília e outra na cidade satélite de Taguatinga. A unidade de portas e janelas localiza-se na cidade de Anápolis, no estado de Goiás. Já a unidade de postes e braços está localizada numa cidade satélite de Brasília. As unidades de portas e janelas e perfilados desenvolvem e fabricam os produtos. Já a unidade de eletroferragens (postes e braços) só realiza montagens.

Os produtos fabricados pela empresa atendem a diversos setores e aplicações construtivas, como construção civil, distribuição, transformadores, iluminação pública e residencial, autopeças, instalações comerciais e industriais, máquinas e implementos agrícolas, indústria moveleira, sistemas de armazenamento, indústria sucroalcooleira e indústria naval.

A competição em cada um dos segmentos é diferenciada: a concorrência se encontra segmentada indo desde pequenas serralherias até empresas de porte maior.

Um diferencial da empresa é a flexibilidade para atender a diferentes demandas por meio de uma vasta gama de produtos, o que implica numa baixa escala de produção. 


\section{Linhas de produtos}

A linha fabricada engloba diferentes tipos de produtos. A unidade de negócios portas e janelas fabrica, além de janelas, venezianas, portas de abrir, portas de correr, porta corta-fogo, maxim-ar e basculantes. A unidade de negócios perfilados fabrica tubos industriais, telhas metálicas, perfis estruturais calandrados e curvados, telhas e cumeeiras, chapas cortadas, dobradas e calandradas, calhas perfiladas e rufos, bem como outros produtos solicitados pela construção civil. Já a unidade de postes e braços atende prioritariamente concessionárias responsáveis pela distribuição de energia elétrica e iluminação pública, fabricando braços para iluminação, postes telecônicos e suportes para luminárias.

\section{Análise e discussão dos resultados}

O trabalho de pesquisa na empresa foi conduzido por meio de pesquisa qualitativa nas modalidades pesquisa documental, observação e estudo de caso. O objetivo dessa primeira fase de pesquisa foi conhecer a história da empresa desde a sua fundação, na década de 1960, e as principais transformações que ocorreram desde então. Nessa etapa foi possível conhecer como a empresa estava estruturada em áreas de negócios e as principais linhas de produtos fabricadas por cada unidade e a utilização de cada produto para atender às mais diversas aplicações construtivas.

Em seguida, foi desenvolvido o estudo de caso nas unidades aplicando-se as técnicas fundamentais de observação e a entrevista. Para a coleta de dados nas fábricas, conforme descrito na metodologia, utilizou-se a pesquisa qualitativa no ambiente natural. Procurou-se identificar o significado que os colaboradores da empresa davam ao processo de implantação do ERP e os seus impactos nas atividades desempenhadas no dia a dia. A abordagem utilizada foi indutiva já que os pesquisadores não partiram de hipóteses estabelecidas a priori, portanto, não se preocuparam em coletar dados que corroborassem ou negassem tais hipóteses. Foi utilizado um instrumento de coleta de dados semiestruturado e realizadas entrevistas com os gestores e demais profissionais envolvidos com planejamento da produção e fabricação. Ao final da entrevista, gravada e transcrita, foram gerados os relatórios narrativos ilustrados com citações, exemplos e descrições do fenômeno fornecidas pelos entrevistados.

Foram entrevistados onze líderes pertencentes às três unidades da empresa. A definição do número de entrevistados considerou que mais entrevistas não melhorariam necessariamente a qualidade da informação ou conduziriam a uma compreensão mais detalhada do fenômeno, já que existe um número limitado de interpretações ou versões da realidade.
As entrevistas iniciaram-se pela Diretora-Executiva da empresa e contemplaram colaboradores de diferentes níveis hierárquicos e funções como gerentes, supervisores, coordenadores e encarregados.

As entrevistas tiveram a duração média de 40 minutos e os roteiros foram customizados a cada respondente em função das atividades desempenhadas por cada um e das informações coletadas junto ao entrevistado anterior. Assim sendo, algumas questões julgadas não pertinentes, em função do cargo ou área de atuação, foram suprimidas nas entrevistas com colaboradores. Da mesma forma, a entrevista com cada liderança possibilitou refinar a abordagem que seria desenvolvida com o próximo entrevistado.

As questões propostas abordaram a implantação do ERP no grupo empresarial, suas repercussões nas unidades de negócio, na produção, nos objetivos de desempenho operacional e na rede de valor de operações.

\section{Impacto da implantação do ERP na empresa}

A primeira parte do roteiro buscou conhecer, de forma geral, a percepção dos entrevistados sobre a repercussão da implantação do ERP na empresa. Foram contempladas questões sobre o que levou a empresa a implantar o $E R P$, principais desafios, principais problemas do período pós-implantação, principais benefícios gerados, o nível de conhecimento dos empregados sobre a empresa no momento da implantação e se o tempo de implantação foi compatível com o estimado.

Com relação ao que motivou a empresa a implantar o $E R P$, foram mencionados pelos entrevistados:

- Melhorias de processos tanto os finalísticos como os de apoio de forma a possibilitar sua rastreabilidade;

- Redução de custos por toda a empresa e processos;

- Necessidade de maior controle e segurança das informações de forma que fossem mais confiáveis e obtidas em tempo real, facilidade para a exportação dessas informações, e redução da sua dispersão propiciada pela integração.

Como principais desafios para a implantação foram mencionados:

- Falta de conhecimento prévio da ferramenta por parte dos colaboradores da empresa, o que dificultou a sua aplicação;

- Desconhecimento da empresa contratada dos processos da contratante para dar suporte adequado à 
implantação bem como ao conhecimento limitado dos consultores sobre a ferramenta. Havia a expectativa da contratante de que todos os consultores da contratada conhecessem todos os módulos, o que não ocorreu;

- Na unidade de portas e janelas, o desafio principal foi migrar de um processo manual para um informatizado. Nas unidades de perfilados, e de postes e braços, em que se verificou a migração entre ferramentas $E R P$, foi mencionada a necessidade de migrar de um sistema para outro e à resistência dos empregados em relação à mudança. Essa resistência se deveu, em parte, à falta de um esforço de conscientização e ao domínio e à segurança com que lidavam com a ferramenta anteriormente utilizada. Foi mencionada também a dificuldade em ter que se envolver com uma nova ferramenta simultaneamente à condução das atividades normais e diárias próprias de cada empregado. Ou seja, a equipe envolvida na implantação não abdicou de suas tarefas rotineiras para se dedicar exclusivamente à implantação da nova ferramenta.

Com relação aos problemas enfrentados no pós-implantação, foram relatadas a falta de parametrização de alguns fatores, grande inter-relação e interdependência entre as áreas, o que muitas vezes travou o andamento da implantação, já que o avanço em um setor é dependente do que é realizado em outro setor. Foi mencionado, ainda, certo desalinhamento entre a produção e o comercial nos primeiros momentos da utilização da ferramenta. Alguns relatórios que eram gerados na ferramenta anteriormente utilizada deixaram de ser gerados na nova ferramenta.

A consequência imediata desse fato foi de que a fábrica produzia e o comercial não "enxergava" essa produção, o que evidencia uma lacuna de integração. Outro fator mencionado é de que a ferramenta é muito "amarrada", isto é, pouco flexível, o que provocou um choque cultural com relação à nova ferramenta, já que os empregados, sobretudo os brasileiros, estavam habituados a um sistema com maior flexibilidade. Também foram relatadas queixas em relação à falta de suporte e apoio de consultoria nos primeiros momentos, nos quais os empregados da contratada ainda não possuíam pleno domínio da ferramenta.

Quanto aos benefícios proporcionados pela adoção do $E R P$, foram percebidos pelos empregados da metalúrgica:

- $\quad$ visão global da empresa;

- maior rastreabilidade e confiabilidade da informação e em particular para o fechamento/conciliação de contas contábeis proporcionada por uma melhor interligação entre as rotinas fiscais e financeiras;

- melhoria na gestão dos processos finalísticos e de suporte;

- maior comprometimento dos empregados com as suas atividades, pois qualquer falha em uma atividade repercute nas demais;

- melhoria no método de custeio da produção, proporcionando maior conhecimento dos custos incorridos na fabricação.

Com relação ao conhecimento prévio que os empregados tinham sobre a empresa, houve consenso de que era satisfatório. Outro ponto em que as percepções foram similares foi quanto ao tempo previsto para a implantação vis a vis o tempo estimado. Todos os entrevistados foram unânimes em afirmar que a implantação durou mais tempo do que o inicialmente previsto.

\section{Impacto na estratégia de produção}

Depois de compreender o efeito da implantação do ERP na empresa e buscando focalizar a produção, objetivo principal do estudo, a entrevista, em um segundo momento, foi conduzida para levantar a percepção dos entrevistados acerca do impacto do ERP nas fábricas e na produção.

Foi investigada a forma com que se deu o levantamento dos processos que antecederam a implantação. Em seguida, procurou-se entender o impacto do ERP nos processos de produção; e avaliou-se os efeitos nos controles da produção, na estratégia de produção de forma abrangente e em particular no planejamento de produção, no processo produtivo, na produtividade, no nível de perdas, desperdício, refugo e retrabalho, na tomada de decisão na produção e na integração com a cadeia de fornecedores.

Com relação ao levantamento dos processos de produção, essa etapa foi facilitada pelo fato de a empresa ser certificada pela ISO 9000. Além das informações proporcionadas pelos documentos da ISO, a empresa contratada conduziu levantamentos junto a alguns empregados para um melhor conhecimento dos processos de produção e das rotinas da metalúrgica. A implantação da ferramenta imprimiu a necessidade de pequenos ajustes em alguns processos. Todavia, as mudanças foram pontuais.

Os controles na produção foi a área sobre a qual os entrevistados apontaram os maiores aprimoramentos, pressupondo-se a precisão das informações impostadas no ERP. As informações do que está sendo produzido são atualmente mais precisas e confiáveis, do que antes da implantação do 
ERP ou na versão anterior de outro fornecedor. A integração dos estoques com o processo produtivo e os inventários realizados ao longo no ano também foram mencionados como ganhos importantes nos controles da produção. Alguns entrevistados manifestaram a expectativa de que o amadurecimento do sistema leve a um maior aprimoramento dos controles.

$\mathrm{Na}$ estratégia, em particular, do planejamento da produção, se observou que as informações confiáveis, proporcionadas pelo $E R P$, tornam o planejamento mais robusto por fornecer informações precisas que propiciam o desenvolvimento de um processo de planejamento adequado à realidade da fábrica, das encomendas, dos estoques de matéria-prima e, consequentemente, uma melhor estratégia de produção. Além disso, auxiliam no planejamento da necessidade de materiais nas fábricas. Com a adoção do $E R P$, as ordens de produção são planejadas e depois programadas no sistema.

No processo produtivo não se observou nenhuma mudança significativa, uma vez que esse é função principalmente da tecnologia empregada e de máquinas e equipamentos. O ERP não comanda nenhum equipamento, embora proporcione informações mais precisas ao operador que deve ajustar a máquina. Apenas foi mencionado, por diversos entrevistados, a maior rastreabilidade da informação no setor de produção e não na fabricação dos produtos em si.

Quanto à produtividade, constatou-se uma ligeira queda no início da implantação decorrente da necessidade de adequar alguns procedimentos. Posteriormente, ela retornou aos níveis normais já que depende, em grande parte, da demanda gerada pelas encomendas que chegam à fábrica, pois a produção é puxada pela comercialização dos produtos.

No que se referem a perdas, desperdícios, refugo e retrabalho, não houve redução na medida em que a perda depende da tecnologia empregada e do que está sendo produzido; todavia, têm-se informações mais precisas da sucata que será gerada no processo produtivo em termos de quantidade/peso e de características da sucata. Outro aspecto importante é que a antecipação das informações acerca do volume de sucata que será gerado no processo permite uma precificação mais precisa da encomenda. Quando o cliente solicita, por exemplo, um determinado volume de um tipo específico de material, avalia-se previamente o volume de sucata que será produzido e esse componente de custo é inserido por ocasião da formação do preço de venda.

A disponibilidade de informações mais precisas e confiáveis proporcionou melhoria na tomada de decisão nas fábricas, haja vista uma maior integração entre a produção propriamente dita e o PPCP (planejamento, programação e controle da produção).
Finalmente, quanto à integração com os fornecedores, foi observada certa dificuldade no início em função das mudanças pelas quais passava a empresa. Superada a dificuldade inicial, hoje se tem uma ligeira melhoria decorrente do fato de que as informações geradas pelo fornecedor entram direto no sistema, ainda que essa integração se limite à entrada da nota fiscal e não do pedido. Existe a expectativa de que essa integração seja aperfeiçoada futuramente, com a adoção de novas e mais sofisticadas versões do $E R P$, por intermédio da integração entre o $E R P$ da empresa e o $E R P$ dos fornecedores.

\section{Impacto nos objetivos de desempenho operacional}

A análise do impacto da adoção do ERP sobre os objetivos de desempenho operacional foi realizada considerando as variáveis: qualidade de processos e produtos, velocidade/ ritmo de produção, confiabilidade dos itens produzidos, flexibilidade na produção para atender encomendas e reflexos nos custos de produção, conforme ilustrado na Figura 3.

Quanto aos reflexos na qualidade dos processos e produtos e na velocidade de produção, não foram relatados ganhos sobretudo porque essas variáveis são determinadas pela qualificação da mão de obra, da tecnologia e dos equipamentos empregados.

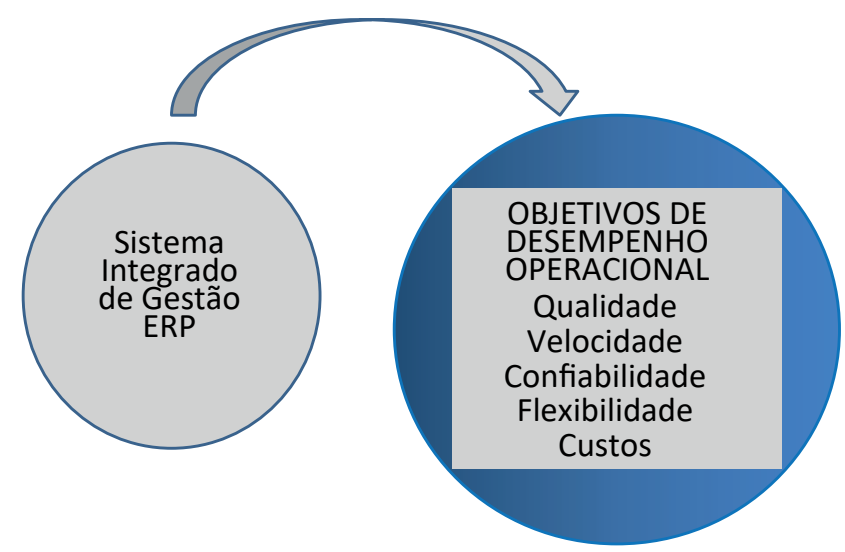

Figura 3. Impacto do ERP nos objetivos de desempenho operacional

Fonte: Os próprios autores

Na fábrica de postes e braços, o processo principal é o de montagem e está relacionado diretamente à qualificação dos trabalhadores envolvidos. Na unidade de negócios de perfilados é utilizado outro sistema que não o $E R P$, para garantir a qualidade de produtos e processos. Esse outro sistema está configurado a partir dos requisitos da norma ISO 9000. 
Um benefício do $E R P$, em termos de qualidade de processos e produtos e velocidade de produção, foi que se reduziu consideravelmente a necessidade de papel circulando nas fábricas, facilitando o processo produtivo e o ritmo de produção, pois a consulta ao sistema, de modo geral, é mais ágil que o manuseio e a busca de informações em relatórios impressos.

Observou-se melhoria no processo de comercialização, dado que o produto acabado aparece mais rapidamente no sistema e essa informação é importante para a área comercial informar ao cliente e agendar a entrega.

No que se refere à confiabilidade dos itens produzidos, o ERP possibilita maior rastreabilidade e melhor tratamento e a análise do pedido item a item ao invés de forma global como era feito anteriormente. Com o ERP o pedido é identificado item a item com a informação de seus respectivos preços e pesos.

A adoção do sistema melhorou a flexibilidade da produção para atender encomendas, já que atualmente se tem mapeado, por meio do ERP, a capacidade de cada equipamento cadastrado e, assim, pode-se melhor alocar os pedidos de forma a aperfeiçoar a capacidade das máquinas. Outro aspecto em relação à flexibilidade é que o ERP prioriza os pedidos independentemente da ordem de chegada.

Uma dificuldade que a empresa enfrenta é que o $E R P$ levou a mudanças nos códigos de produtos, matérias-primas e componentes. Anteriormente, os códigos levavam em consideração famílias de materiais e agora são apenas números sequenciais.

Com relação aos custos de produção, ainda não foram identificados ganhos. Todavia, houve depoimentos no sentido de que hoje eles são mais bem calculados. Quando se implanta uma ordem de produção, já se tem um custo estimado, e quando essa é finalizada é possível comparar o custo real com o custo planejado. No caso de geração de sucata, ao atender um pedido, a informação precisa possibilitar que a precificação seja refinada incorporando esse item de custo. Em outras palavras, antes de concretizar a venda, o vendedor já tem as dimensões do produto e informação sobre as perdas que serão geradas no processo produtivo, o que permite que esse valor seja repassado ao cliente que solicitou a encomenda.

Um dos entrevistados chamou a atenção para o fato de que o tempo da mão de obra hoje é mais bem utilizado. No $E R P$ anterior era informado apenas o momento do início da produção e o momento em que a mesa era encerrada. Atualmente, a ordem de produção já possui um tempo estimado para a fabricação do produto, o que possibilita um melhor controle da supervisão da operação e do trabalho do empregado da produção a partir desse referencial. Caso a fabricação demore mais tempo do que o inicialmente previsto, o supervisor da produção pode verificar o que pode estar acontecendo.

Ainda com relação aos custos de produção, não se pode ignorar que eles são determinados, em grande medida, pelos custos de energia elétrica, de mão de obra e da matéria-prima, nesse caso, o aço.

\section{CONCLUSÃO}

Com base no estudo foi possível constatar que para a implantação do $E R P$, que contou com o comprometimento da alta direção, foram identificados os usuários-chave, foi definido um gerente para o projeto, os treinamentos foram planejados e realizados, os papéis na implantação foram definidos e o sistema foi customizado em função da realidade da empresa, conforme recomendado por Padilha et al. (2005).

A adoção do ERP trouxe melhorias para a operação da metalúrgica, como preconizado por Chatzoglou et al. (2016) e Laudon e Laudon (2007), sobretudo na confiabilidade e rastreabilidade da informação. A implantação foi determinada pela necessidade de integrar muitas informações que se encontravam dispersas nos diversos sistemas, dificultando o resgate dessas informações de forma ágil, bem como considerou os problemas decorrentes de informações divergentes entre os vários sistemas e bases de dados. O ERP possibilitou integrar as informações dos diversos setores da empresa em uma única base de dados, o que aprimorou o processo de planejamento da produção e consequentemente da elaboração das estratégias de produção por atender a necessidades de informações confiáveis e em tempo real. Ou seja, a adoção do ERP aprimorou as operações da empresa, reduzindo perdas e mitigando o risco da operação, já que possibilitou integrar informações das diversas unidades de negócios assegurando a sua confiabilidade e disponibilidade e o acesso em tempo real para a tomada de decisão.

Como mencionado por Cao e Dowlatshahi (2005), o estudo permitiu identificar vínculos importantes entre a estratégia de produção e a orientação estratégica de sistemas de informação e a correlação entre o desempenho da metalúrgica e os sistemas de informação (Byrd et al., 2006; Chan; Reich, 2007).

Conforme abordado por Sabherwal e Chan (2001) e Chan (2002), este estudo identificou a integração entre a orientação estratégica de sistemas de informação e as decisões em termos de operações, da mesma forma que entre a estratégia de negócios e a estratégia de informações no desempenho do negócio. 
As questões culturais envolvidas na implantação do ERP também puderam ser identificadas, com antecipado por Yusuf et al. (2004), quando estudaram a implementação do ERP na Rolls-Royce, utilizando a metodologia do estudo de caso, focando principalmente no projeto de implementação, nos aspectos culturais envolvidos e nos problemas técnicos da implementação. O relacionamento da implantação do ERP com a estratégia e com aspectos culturais envolvidos em alinhar a implementação do ERP a produtos e processos também foi abordado.

Uma lacuna que foi possível observar se deveu ao dimensionamento de prazos e expectativas, que foram subestimados conforme mencionado por Ghosh (2012).

Com relação aos controles na produção, os entrevistados apontaram os maiores aprimoramentos condicionados à precisão das informações impostadas no sistema. As informações sobre o que está sendo produzido se tornaram mais precisas e confiáveis quando em comparação ao momento anterior à implantação do $E R P$ ou e à versão de outro fornecedor.

Especificamente com relação aos objetivos de desempenho operacional, Yen e Sheu (2004) e Yusuf et al. (2004) observaram:

Um benefício do ERP em termos de qualidade de processos e produtos e velocidade de produção foi que se reduziu consideravelmente a necessidade de papel circulando nas fábricas, facilitando o processo produtivo e elevando o ritmo de produção, uma vez que a consulta ao sistema é mais ágil que o manuseio e a busca de informações em relatórios impressos:

No que se refere à confiabilidade dos itens produzidos, o ERP possibilita maior rastreabilidade e melhor tratamento e a análise do pedido item a item ao invés da forma global como era feito anteriormente. Com o ERP, o pedido é identificado com a informação de seus respectivos preços e pesos;

A adoção do ERP melhorou a flexibilidade da produção para atender encomendas, já que atualmente se tem mapeado a capacidade de cada equipamento cadastrado e, assim, pode-se melhor alocar os pedidos de forma a aperfeiçoar a capacidade das máquinas;

Com relação aos custos de produção, ainda não foram identificadas informações que possibilitem concluir que houve ganho, todavia, constatou-se que atualmente eles são calculados com maior precisão. Quando se implanta uma ordem de produção, por exemplo, já se tem o custo estimado, e quando essa é finalizada é possível comparar o custo real com o custo planejado. No caso de geração de sucata, ao atender um pedido, a informação confiável possibilita que a precificação do produto ocorra incorporando esse item de custo ao preço final.

Adicionalmente, observou-se que a disponibilidade da mão de obra passou a ser mais bem utilizada. No sistema anterior, no caso das unidades que já possuíam ERP de outro fornecedor, era informado apenas o momento do início da produção e o momento em que a mesa era encerrada. Atualmente, a ordem de produção já apresenta o tempo estimado para a fabricação do produto, possibilitando um melhor controle da supervisão da operação e do trabalho do empregado da produção a partir desse referencial. Caso a fabricação demore mais tempo do que o inicialmente previsto, o supervisor da produção pode verificar o que pode estar acontecendo.

Finalmente, com relação ao suprimento das fábricas, constatou-se, ouvidos os entrevistados, ganhos importantes que podem se intensificar com uma maior integração entre o ERP da metalúrgica e de seus fornecedores. Melhorias incrementais foram constatadas, como mencionadas anteriormente, na entrada da nota que é feita diretamente no sistema com o registro de mais detalhes e informações.

Com base nas análises realizadas, o objetivo do estudo foi alcançado ao avaliar o impacto da implementação do ERP na estratégia de produção na empresa.

\section{REFERÊNCIAS}

Amoako-Gyampah, K., Boye, S. S. Operations strategy in an emerging economy: the case of the Ghanaian manufacturing industry. Journal of Operations Management, 19, 59-79. https://doi.org/10.1016/S0272-6963(00)00046-2

Anderson, J. C., Cleveland, G., Schoroeder, R. G. 1989. Operations strategy: a literature review. Journal of Operations Management, 8, 133-158. https://doi.org/10.1016/02726963(89)90016-8

Barth, C., Koch, S. 2019. Critical success factors in ERP upgrade projects. Industrial Management \& Data Systems, 119, 656 675. https://doi.org/10.1108/IMDS-01-2018-0016

Bowersox, D.J., Closs, D.J., Hall, C.T. 1998. Beyond ERP - The storm before the calm. Supply Chain Management Review, 1, 28-36.

Byrd, A.T., Lewis, B.R., Bryan, R.W. 2006. The leveraging influence of strategic alignment on IT investment: an empirical examination. Information \& Management, 43, 308-321. https://doi.org/10.1016/j.im.2005.07.002

Cao, Q., Dowlatshahi, S. 2005. The impact of alignment between virtual enterprise and information technology on business performance in an agile manufacturing environment. 
Journal of Operations Management, 23, 531-550. https://doi. org/10.1016/j.jom.2004.10.010

Chan, Y., Reich, B. H. 2007. IT alignment: what have we learned? Journal of Information Technology, 22, 297-315. https://doi.org/10.1057/palgrave.jit.2000109

Chan, Y.E. 2002. Why haven't we mastered alignment? The importance of the informal organization structure. MIS Quarterly Executive 1, 97-112.

Chatzoglou, P., Chatzoudes, D., Fragidis, L. et al. 2016. Critical success factors for ERP implementation in SMEs. Proceedings of the Federated Conference on Computer Science and Information Systems, 8, 1243-1252. https://doi. org/10.15439/2016F37

Chen, H. H., Chen, S. C., Tsai, L. H. 2009. A study of successful ERP - from the organization fit perspective. Systemics, Cybernetics and Informatics, 7, 8-16.

Cohen, M. A., Lee, H. L. 1985. Manufacturing Strategy - Concepts and Methods. In: Kleindorfer, P. R. The Management of Productivity and Technology in Manufacturing. New York: Plenum Press, cap. 5, p. 153-186.

Comissão Econômica para a América Latina e o Caribe - CEPAL, Instituto de Pesquisa Econômica Aplicada - IPEA. 2018. Avaliação de desempenho do Brasil mais produtivo. Brasília, CEPAL, IPEA.

Dantes, G. R., Hasibuan, Z. A. 2011. The impact of enterprise resource planning (ERP) system implementation on organization: case study ERP implementation in Indonesia. IBIMA Business Review, 2011, 210664. https://doi. org/10.5171/2011.210664

Davenport, T. H. 1998. Putting the enterprise into the enterprise system. Harvard Business Review 76, 121-131.

Esmaeel, R.I., Zakuan, N., Jamal, H. N. M, et al. 2018. Understanding of business performance from the perspective of manufacturing strategies: fit manufacturing and overall equipment effectiveness. Procedia Manufacturing, 22, 9981006. https://doi.org/10.1016/j.promfg.2018.03.142

Ferreira, D.D., Alves, J.M., Araújo Júnior, L. S., et al. 2010. Análise do processo de migração do sistema MRP legado para o MRP II: um estudo de caso na indústria aeronáutica. Simpósio de Pesquisa Operacional e Logística da Marinha, 12-13 ago. 2010, Rio de Janeiro.

Garvin, D. A. 1993. Manufacturing Strategy Planning. California Management Review, 35, 85-106. https://doi. org/10.2307/41166756

Ghosh, R. A. 2012. A comprehensive study on ERP failures stressing on reluctance to change as a cause of failure. Journal of Marketing and Management, 3, 123-134.

Hammer, M., Stanton, S. 1999. How process enterprises really work. Harvard Business Review, 77, 108-118.
Hill, T. 2005. Operations management. 2nd ed. Macmillan, Basingstoke.

Huang, T., Yokota, A. 2019. Inventing a business-ERP alignment assessment model through three Japanese companies. Business Process Management Journal, 25, 738-756. https:// doi-org.ez54.periodicos.capes.gov.br/10.1108/BPMJ-032017-0068

Jacobs, F.R., Whybark, D.C. 2000. Why ERP A Primer on SAP Implementation. Irwin/McGraw-Hill, NewYork.

Junior, C. C. 2008. Sistemas Integrados de Gestão ERP. 3. Ed. Curitiba: IBPEX.

Laudon, J. P., Laudon, K. C. 2007. Sistemas de informações gerenciais. 7 ed. São Paulo: Pearson.

Lauras, M., Marques, G., Gourc, D. 2010. Towards a multi-dimensional project performance measurement system. Decision Support Systems, 48, 342-353. https://doi.org/10.1016/j. dss.2009.09.002

Mazzoti, A., Alves, J. 2006. Usos e abusos do estudo de caso. Cadernos de Pesquisa, 36, 637-651. https://doi.org/10.1590/ S0100-15742006000300007

Nudurupati, S.S., Bititci, U.S., Kumar, V. et al. 2011. State of the art literature review on performance measurement. Computers \& Industrial Engineering, 60, 279-290. https://doi. org/10.1016/j.cie.2010.11.010

Okoshi, C.Y, Lima, E.P., Costa, S.E.G. 2019. Performance cause and effect studies: Analyzing high performance manufacturing companies. International Journal of Production Economics, 210, 27-41. https://doi.org/10.1016/j.ijpe.2019.01.003

Oleskow, J. Pawlewski, P., Fertsch, M. 2002. Limitations and performance of MRP II/ERP systems - Significant contribuition of Al techniques, 19 th International Conference on Production Research.

Padilha, T., Cabral C., Marins, F. A. S. 2005. Sistemas ERP: características, custos e tendências. Revista Produção, 15, 102113.

Paiva, E. L., Carvalho Jr. J. M., Fensterseifer, J. E. 2009. Estratégia de produção e de operações. 2a ed. Porto Alegre: Bookman.

Peng, G.C., Nunes, M. 2017. Establishing an evidence-based 9D evaluation approach for ERP post-implementation. Industrial Management \& Data Systems, 117, 398-424. https://doi. org/10.1108/IMDS-03-2016-0087

Rashid M.A., Hossain, L., Patrick, J.D. 2002. The evolution of ERP systems: a historical perspective, Idea Group Publishing.

Rayner, N. 2014. Survey analysis: adoption of cloud ERP, 2013 through 2023. Gartner Group Inc., Connecticut.

Sabherwal, R., Chan, Y.E. 2001. Alignment between business and IS strategies: a study of prospectors, analysers, and de- 
fenders. Information Systems Research, 12, 112-134. https:// doi.org/10.1287/isre.12.1.11.9714

Scheckenbach, T., Zhao, F., Allard, E., et al. 2014. Issues of ERP upgrade in public sectors: a case study. In: Kurosu, M. (Ed.). Human-Computer Interaction. 16th International Conference, $\mathrm{HCl}$ International 2014, Heraklion, Crete, Greece, June 22-27, 2014, Proceedings, Part III, pp. 754-763.

Skinner, W. 1969. Manufacturing-missing link in corporate strategy. Harvard Business Review, 47, 136-145.

Slack, N. 2005. Operations strategy: Will it ever realize its potential? Gestão e Produção, 12, 323-332. https://doi. org/10.1590/S0104-530X2005000300004

Slack, N., Chambers, S., Johnston R. 2010. Operations Management, FT Prentice Hall.

Soh, C., Kien, S.S., Tay-Yap, J. 2000. Enterprise Resource Planning: Cultural fits and misfits: Is ERP a universal solution? Communications of the ACM, 43, 47-51. https://doi. org/10.1145/332051.332070
Swamidass, P. M., Newell, W. T. 1987. Manufacturing strategy, environmental uncertainly and performance: a path analytical model. Management Science, 33, 509-524.

Valipour, H., Moradi J. Fatheh, M. H. 2012. The impact of Enterprise Resource Planning (ERP) on the internal controls case study: Esfahan Steel Company. European Journal of Social Sciences, 28, 228-238.

Yen, H. R., Sheu, C. 2004. Aligning ERP implementation with competitive priorities of manufacturing firms: an exploratory study. International Journal of Production Economics, 92, 207220. https://doi.org/10.1016/j.ijpe.2003.08.014

Yin, R. K. 2005. Estudo de caso: planejamento e método. 2. ed. São Paulo: Bookman.

Yusuf, Y., Gunasekaran, A. E, Abthorpe, M. S. 2004. Enterprise Information systems project implementation: a case study of ERP in Rolls-Royce. International Journal of Production Economics, 87, 251-266. https://doi.org/10.1016/j. ijpe.2003.10.004

Recebido: 18 jun. 2020

Aprovado: 30 jun. 2020

DOI: 10.20985/1980-5160.2020.v15n2.1652

Como citar: Affonso Neto, A., Neumann, C. (2020). Implementação do Enterprise Resource Planning em uma metalúrgica com linhas de produtos diferenciadas. Revista S\&G 15, 2, 143-155. https://revistasg.emnuvens.com.br/ sg/article/view/1652 